\title{
钯催化的二甲亚砜对炔酰胺的氧化反应: $\alpha$-酩酰胺衍生物的高效合成方法
}

孟团结冯翠兰刘澜涛* 王 涛许凯赵文献*

(商丘师范学院化学化工学院 药物绿色合成河南省工程实验室 商丘 476000)

\begin{abstract}
摘要 以钯为催化剂, 利用便宜易得的二甲亚砜为氧化剂和溶剂, 通过炔酰胺的氧化反应, 高效合成了一系列 $\alpha$-酮酰 胺衍生物. 通过 ${ }^{1} \mathrm{H} N M R,{ }^{13} \mathrm{C} N \mathrm{NR}, \mathrm{IR}$ 和 HRMS 对产物结构进行了表征. 该方法具有反应条件温和、操作简单、原料 易得、反应迅速的特点.
\end{abstract}

关键词 二甲亚砜; 炔酰胺; $\alpha$-酮酰胺; 合成; 氧化

\section{Palladium Catalyzed Oxidation of Ynamides Using Dimethyl Sulfox- ide as Oxidant: A Facile Way to Synthesize $\alpha$-Ketoamide Derivatives}

\author{
Meng, Tuanjie Feng, Cuilan Liu, Lantao* Wang, Tao Xu, Kai Zhao, Wenxian* \\ (Engineering Laboratory of Henan Province for Green Synthesis of Medicine, College of Chemistry and Chemical \\ Engineering, Shangqiu Normal University, Shangqiu 476000)
}

\begin{abstract}
A series of $\alpha$-ketoamide derivatives were obtained from the palladium catalyzed oxidation of ynamides using cheap and readily available dimethyl sulfoxide as oxidant and solvent under room temperature. The structures of all products were characterized by ${ }^{1} \mathrm{H}$ NMR, ${ }^{13} \mathrm{C}$ NMR, IR and HRMS. This protocol has some distinct advantages of mild conditions, simple work-up, readily available starting materials and fast reaction rate.
\end{abstract}

Keywords dimethyl sulfoxide; ynamides; $\alpha$-ketoamides; synthesis; oxidation

$\alpha$-酮酰胺化合物是一类重要的有机合成中间体，并 且用于疮疾蛋白酶抑制剂 ${ }^{[1]}$ 、钙蛋白酶抑制剂 ${ }^{[2]}$ 、诺如 病毒蛋白酶抑制剂 ${ }^{[3]}$ 、登革热病毒蛋白酶抑制剂 ${ }^{[4]}$ 、组 织蛋白酶 $\mathrm{S}$ 抑制剂 ${ }^{[5]}$ 、胰脂肪酶抑制剂 ${ }^{[6]}$ 的分子结构设 计中. 治疗丙型肝炎的新药 Boceprevir ${ }^{[7]}$ 和 Telaprevir $^{[8]}$ 及治疗湿疹的药物 Tacrolimus (FK506) ${ }^{[0]}$ 均含有 $\alpha$-酮酰 胺结构单元(图 1).

由于 $\alpha$-酮酰胺衍生物具有广泛的生物活性，其合成 方法已成为研究的热点. 此类化合物的传统的合成方法 是 $\alpha$-酮酸的酰胺化反应 ${ }^{[10]}$. 通过芳基甲基酮 ${ }^{[11]}$ 、芳基乙 炔 ${ }^{[12]}$ 、芳基乙烯 ${ }^{[13]}$ 、芳基乙烷 ${ }^{[14]} 、 \alpha$-羰基醛 ${ }^{[15]}$ 、芳基乙 醛 ${ }^{\left[{ }^{[16]}\right.}$ 的氧化酰胺化也是一类常用的方法. 另外芳基 碘 ${ }^{[17]}$ 或吲哚 ${ }^{[18]}$ 的双羰化酰胺化也可构建 $\alpha$-酮酰胺衍生
物. 近年来, 炔酰胺的氧化已经成为构建 $\alpha$-酩酰胺的重 要方法. 2008 年 Al-Rashid 等 ${ }^{[19]}$ 报道了炔酰胺可被 $\mathrm{RuO}_{4}$ 或过氧丙酮(DMDO)氧化体系氧化高产率地得到 $\alpha$-酮酰 胺, 但这两种氧化剂都需要大大过量, 且不易保存. 2014 年朱红军 ${ }^{[20]}$ 和 Iwasawa ${ }^{[21]}$ 研究小组分别报道了过 量 NIS 或碘的促进的炔酰胺氧化反应合成 $\alpha$-酮酰胺. 在 金的催化下利用二苯亚砜为氧化剂氧化炔酰胺也可得 到 $\alpha$-酮酰胺 ${ }^{[22]}$. 但这些方法存在催化剂较昂贵或需使 用过量的促进剂 NIS 及碘等缺点. 虽然已有众多的方法 合成 $\alpha$-酮酰胺类化合物, 但寻到反应条件温和操作更加 简单的方法仍然是一个挑战. 二甲亚砜不仅是常用的有 机溶剂，近年来由于其廉价易得且操作简便，作为氧化 剂得到较大发展 ${ }^{[23]}$. 鉴于此, 本文报道在室温条件钯催

\footnotetext{
*E-mail: liult05@iccas.ac.cn, zhwx2595126@163.com

Received January 3, 2016; revised February 4, 2016; published online February 18, 2016.

Project supported by the National Natural Science Foundation of China (Nos. U1204204, 21172139, 21202095), the Science and Technology Key Project of Henan Province (No. 142102210635), and the Program for University Key Young Teachers of Henan Province (No. 2015GGJS-126).

国家自然科学基金(Nos. U1204204，21172139，21202095)、河南省科技攻关项目(No. 142102210635)和河南省高等学校青年骨干教师(No. 2015GGJS-126)资助项目.
} 
化下以二甲亚砜为氧化剂通过炔酰胺的氧化反应高产 率地合成 $\alpha$-酮酰胺的新方法.

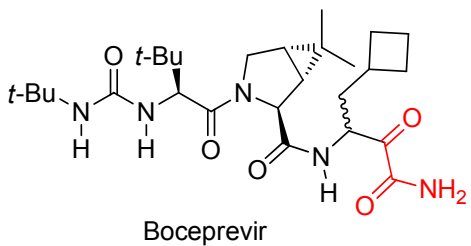<smiles>CCCC(NC(=O)[C@H]1[C@H]2CCC[C@H]2CN1C(=O)[C@H](C)NC(=O)[C@H](NC(=O)c1cnccn1)C1CCCCC1)C(=O)NC1CC1</smiles>

Telaprevir

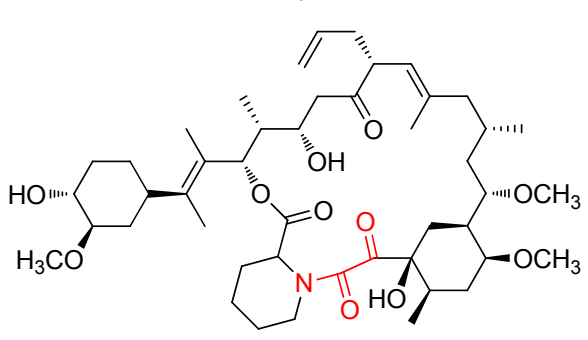

Tacrolimus

图 1 三个含 $\alpha$-酮酰胺结构的药物

Figure 1 Three pharmaceuticals containing $\alpha$-ketoamide moieties

\section{1 结果与讨论}

\section{1 反应条件考察}

首先，以 $N$-芐基- $N$-苯乙炔基对甲苯磺酰胺(1a) 与 二甲亚砜(DMSO)为模型反应底物, 考察了催化剂、反应 温度、反应时间对反应结果的影响, 实验结果见表 1 . 仅 在醋酸钯的催化下无论是室温或加热均只能得到痕量 的产物(表 1, Entries 1,2). 当加入二价铜作为共催化剂 时，反应产率大幅度提高至 $60 \%$ 以上(表 1, Entries 3 5). 仅以二价铜为催化剂时未发现目标产物的生成(表 1 , Entries 6 8). 此结果与 2012 年吴养洁研究组 ${ }^{[2]}$ 报道的 结果相似. 当催化剂的用量增加至 $10 \mathrm{~mol} \%$ 时, 产率提 高至 92\%(表 1, Entry 9). 在氞气的保护下该反应能顺利 地进行, 并高产率地得到目标产物(表 1, Entry 10). 当将 溶剂由 DMSO 改为四氢呋喃(THF)、 $N, N$-二甲基甲酰胺 (DMF)或 1,2-二氯乙烷(DCE)时根本无目标产物的生成 (表 1, Entries 15 17), 此结果说明氧化炔酰胺的是 DMSO 而不是空气中的氧气. 我们还考察了不同钯源对 反应的影响, 以醋酸钯的催化效果最好(表 1, Entries 9
13). 当反应在冰水浴中进行时反应收率大幅度降低(表 1, Entry 14)。最终我们确定反应条件是：催化剂 $\mathrm{Pd}(\mathrm{OAc})_{2} / \mathrm{CuCl}_{2}$ 的用量为 $10 \mathrm{~mol} \%$, DMSO 既作溶剂又 作氧化剂, 在室温空气气氛下反应 $2 \mathrm{~h}$.

表 1 反应条件优化 ${ }^{a}$

Table 1 Reaction conditions optimization

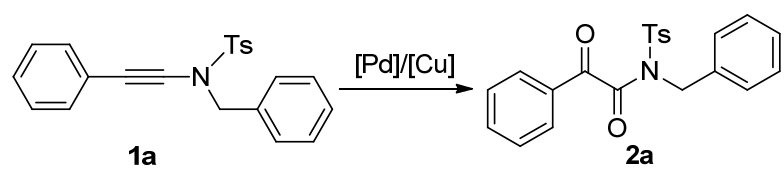

\begin{tabular}{clccc}
\hline Entry & \multicolumn{1}{c}{ Catalyst $(\mathrm{mol} \%)$} & Solvent & $T /{ }^{\circ} \mathrm{C}$ & Yield/\% \\
\hline 1 & $\mathrm{Pd}(\mathrm{OAc})_{2}(5)$ & DMSO & r.t. & Trace \\
2 & $\mathrm{Pd}(\mathrm{OAc})_{2}(5)$ & DMSO & 80 & Trace \\
3 & $\mathrm{Pd}(\mathrm{OAc})_{2} / \mathrm{CuCl}_{2}(5)$ & DMSO & r.t. & 65 \\
4 & $\mathrm{Pd}(\mathrm{OAc})_{2} / \mathrm{CuBr}_{2}(5)$ & DMSO & r.t. & 65 \\
5 & $\mathrm{Pd}(\mathrm{OAc})_{2} / \mathrm{Cu}(\mathrm{OAc})_{2}(5)$ & DMSO & r.t. & 62 \\
6 & $\mathrm{CuCl}_{2}(5)$ & DMSO & r.t. & 0 \\
7 & $\mathrm{CuBr}(5)$ & DMSO & r.t. & 0 \\
8 & $\mathrm{Cu}(\mathrm{OAc})_{2}(5)$ & DMSO & r.t. & 0 \\
9 & $\mathrm{Pd}(\mathrm{OAc})_{2} / \mathrm{CuCl}_{2}(10)$ & DMSO & r.t. & 92 \\
$10^{b}$ & $\mathrm{Pd}(\mathrm{OAc})_{2} / \mathrm{CuCl}_{2}(10)$ & DMSO & r.t. & 90 \\
11 & $\mathrm{PdCl} / \mathrm{CuCl}_{2}(10)$ & DMSO & r.t. & 85 \\
12 & $\mathrm{Pd}\left(\mathrm{PPh}_{3}\right)_{2} \mathrm{Cl}_{2} / \mathrm{CuCl}_{2}(10)$ & DMSO & r.t. & 77 \\
13 & $\mathrm{Pd}\left(\mathrm{dba}_{2} / \mathrm{CuCl}_{2}(10)\right.$ & DMSO & r.t. & 74 \\
14 & $\mathrm{Pd}(\mathrm{OAc})_{2} / \mathrm{CuCl}_{2}(10)$ & DMSO & 0 & 44 \\
15 & $\mathrm{Pd}(\mathrm{OAc})_{2} / \mathrm{CuCl}_{2}(10)$ & THF & r.t. & 0 \\
16 & $\mathrm{Pd}(\mathrm{OAc})_{2} / \mathrm{CuCl}_{2}(10)$ & DMF & r.t. & 0 \\
17 & $\mathrm{Pd}(\mathrm{OAc})_{2} / \mathrm{CuCl}_{2}(10)$ & DCE & r.t. & 0 \\
\hline
\end{tabular}

${ }^{a}$ All reactions were carried out in air atmosphere, ynamide $(1.0 \mathrm{mmol})$ in $2 \mathrm{~mL}$ solvent. ${ }^{b}$ Under the argon atmosphere.

\section{2 底物的拓展}

在上述得到的较佳反应条件下，我们考察了底物炔 酰胺的适用范围，具体见表 2. 实验表明此反应具有较 好的官能团兼容性. 炔酰胺中氮原子上 $\mathrm{R}^{1}$ 是对甲苯磺 酰基 $\mathrm{Ts}$ (表 2, 2a $\sim 2 \mathbf{f}$ )与甲磺酰基 $\mathrm{Ms}$ (表 2, 2h $\sim 2 \mathbf{n}$ )时反 应都能顺利进行, 且高产率地生成 $\alpha$-酮酰胺衍生物. 当 $\mathrm{R}^{1}$ 为 $\mathrm{Ms}$ 时比 $\mathrm{Ts}$ 时产率略低(表 $2, \mathbf{2 a}$ vs. $\mathbf{2 i}, \mathbf{2 b}$ vs. $\mathbf{2 h}, \mathbf{2 f}$ vs. $\mathbf{2 j}$ ), 这可能是由于 $M s$ 的吸电子能力较强, 减弱氮原 子向炔键供电子能力使炔键较难被氧化. 当 $\mathrm{R}^{2}$ 是茮基、 脂肪烃基、苯基取代基时都可得到高产率的目标产物, 且芐基上的甲氧基、溴、氯、氟、甲基等取代基对反应 产率无明显影响. 当 $\mathrm{R}^{2}$ 是烯丙基时产率仅为 $43 \%$ (表 2, 2d). 这是因为在钯的作用下烯丙基较易离去而形成烯 丙基钯中间体 ${ }^{[25]}$. 当利用 3-苯乙炔基噁唑啉-2-酮为炔 酰胺底物仅能得到中等产物的收率(表 $2, \mathbf{2 g}$ ). 当使用叔 丁氧羰基 Boc 保护的炔胺为底物时仅得到 28\%的其自 身环化产物啞唑酮 ${ }^{[26]}$, 没有得到其氧化产物 $\alpha$-酮酰胺 (表 2, 2o, Eq. 1). 
表 2 钯催化的炔酰胺氧化反应 ${ }^{a, b}$

Table 2 Palladium catalyzed oxidation of ynamides

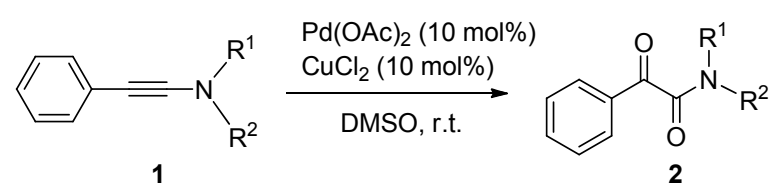<smiles>CN(Cc1ccccc1)C(=O)C(=O)c1ccccc1</smiles>

2a $92 \%$<smiles>C=CCN([13CH3])C(=O)C(=O)c1ccccc1</smiles>

2d $43 \%$<smiles>O=C(C(=O)N1CCOC1=O)c1ccccc1</smiles>

$\operatorname{2g} 61 \%$<smiles>CS(=O)(=O)N(Cc1ccc(F)cc1)C(=O)C(=O)c1ccccc1</smiles>

2j $84 \%$<smiles>COc1ccc(N(C(=O)C(=O)c2ccccc2)S(C)(=O)=O)cc1</smiles>

2m $80 \%$<smiles>COc1ccc(CN([13CH3])C(=O)C(=O)c2ccccc2)cc1</smiles>

2b 93\%<smiles>[3H]N(CCCC)C(=O)C(=O)c1ccccc1</smiles>

2e $90 \%$<smiles>COc1ccc(CN(C(=O)C(=O)c2ccccc2)S(C)(=O)=O)cc1</smiles>

2h $84 \%$<smiles>Cc1ccc(CN(C(=O)C(=O)c2ccccc2)C(C)(C)C)cc1</smiles>

2k $86 \%$<smiles>Cc1ccc(N(C(=O)C(=O)c2ccccc2)C(C)(C)C)cc1</smiles>

2n $77 \%$<smiles>CN(Cc1ccccc1Br)C(=O)C(=O)c1ccccc1</smiles>

2c $89 \%$<smiles>[13CH3]N(Cc1ccc(F)cc1)C(=O)C(=O)c1ccccc1</smiles>

2f $87 \%$<smiles>CS(=O)(=O)N(Cc1ccccc1)C(=O)C(=O)c1ccccc1</smiles>

2i $88 \%$<smiles>CS(=O)(=O)N(Cc1ccccc1Cl)C(=O)C(=O)c1ccccc1</smiles>

2l $82 \%$<smiles>Cc1ccc(N(C(=O)OC(C)(C)C)C(=O)C(=O)c2ccccc2)cc1</smiles>

$200 \%$

${ }^{a}$ All reactions were carried out at room temperature in air atmosphere. Reaction condition: ynamide $(1.0 \mathrm{mmol}), \mathrm{Pd}(\mathrm{OAc})_{2}(0.1 \mathrm{mmol}), \mathrm{CuCl}{ }_{2}(0.1 \mathrm{mmol})$, DMSO $(2 \mathrm{~mL}) .{ }^{b}$ Isolated yield.

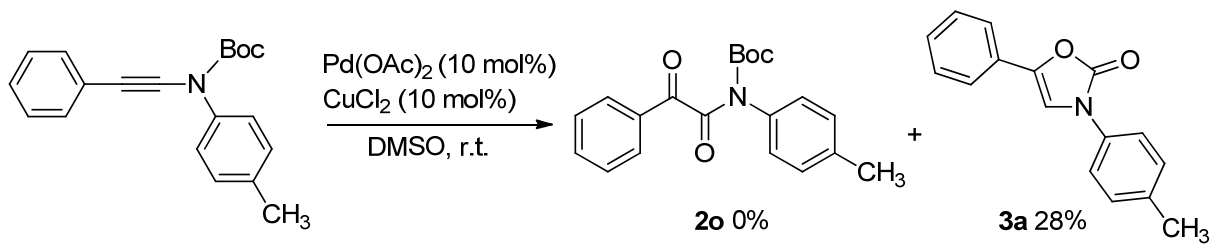

\section{3 可能的反应机理}

根据上述实验结果及文献报道 ${ }^{[24]}$, 我们提出图 2 所 示的可能的反应机理. 首先二价钯与炔酰胺 1 中碳碳参 键络合生成中间体 $\mathbf{A}, \mathbf{A}$ 中氮原子上未成键电子对迁移 到氮与 $\alpha$ 碳原子之间形成烯酮亚胺正离子中间体 $\mathbf{B}^{[27]}$, DMSO 中的氧原子作为亲核试剂的亲核中心进攻烯酮 亚胺正离子中间体 $\mathbf{B}$ 的 $\alpha$ 碳得到中间体 $\mathbf{C}$, 另一分子的 DMSO 进攻 $\beta$ 碳同时离去一分子二甲硫醚得到中间体 $\mathbf{D}, \mathbf{D}$ 发生 $\beta$ 消除生成目标产物 $\alpha$-酮酰胺, 同时释放出二 价钯进行下一个催化循环。

\section{2 结论}

在醋酸钯的催化下利用便宜易得的二甲亚砜为氧 化剂和溶剂, 无需惰性气体的保护在室温条件下实现了 炔酰胺的氧化, 高产率地得到 $\alpha$-酮酰胺衍生物, 为 $\alpha$-酮 酰胺的合成提供了一种新方法. 该方法具有反应条件温 和、操作简单、原料易得、反应迅速的优点. 我们考查 了底物的适用范围，当底物炔酰胺中氮原子上的吸电子 基团是 $\mathrm{Ts}$ 或 $\mathrm{Ms}$ 时适用于该反应，当是 Boc 基团时不适 用. 


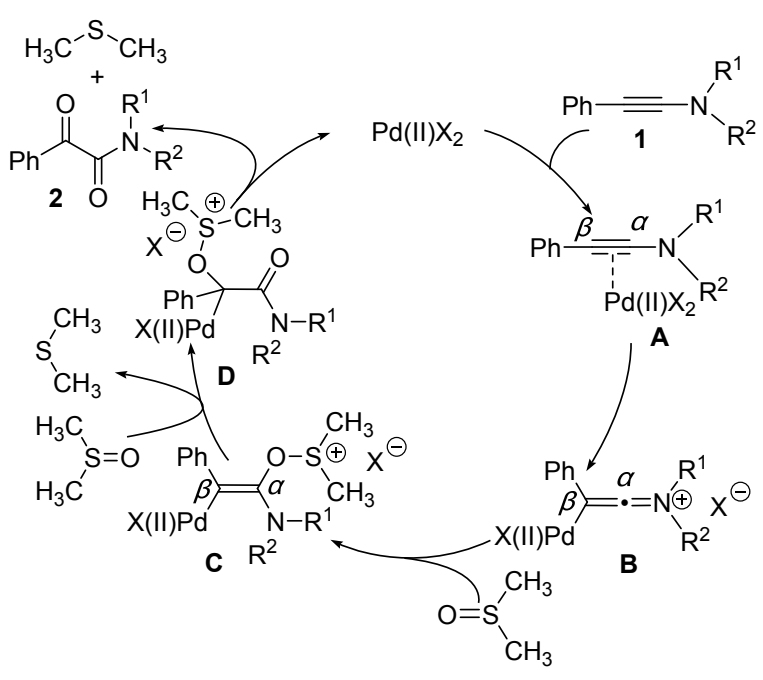

图 2 可能的反应机理

Figure 2 Proposed reaction mechanism

\section{3 实验部分}

\section{1 仪器与试剂}

核磁共振氢谱 ${ }^{1} \mathrm{H}$ NMR $(400 \mathrm{MHz})$ 和 ${ }^{13} \mathrm{C}$ NMR $(100$ $\mathrm{MHz}$ ) 采用 Bruker AV 400 核磁共振仪测定, 所用溶剂为 $\mathrm{CDCl}_{3}$, TMS 为内标; 熔点测定仪采用巩义予华仪器有 限责任公司生产的 XT-5 型熔点测定仪(温度未经校正); 高分辨质谱使用 Agilent LC-MSD-Trap-XCT 仪器测定; 红外光谱用美国 VARIAN 公司 Scimitar 2000 型光谱仪 测定 $(\mathrm{KBr}$ 压片); 柱层析使用 200 300 目硅胶, 展开剂 为石油醚 $\left(60 \sim 90{ }^{\circ} \mathrm{C}\right)$ 和乙酸乙酯. 底物炔酰胺 1 参考文 献方法合成 ${ }^{[19-22]}$; 实验所用试剂均为市售 $\mathrm{AR}$ 试剂.

\section{2 实验方法}

在 $25 \mathrm{~mL}$ 反应管中加入 $1.0 \mathrm{mmol}$ 炔酰胺, $0.1 \mathrm{mmol}$ $\mathrm{Pd}(\mathrm{OAc})_{2}, 0.1 \mathrm{mmol} \mathrm{CuCl}_{2}, 2 \mathrm{~mL} \mathrm{DMSO}$, 室温反应 $2 \mathrm{~h}$, TLC 检测反应完全后, 向反应体系中加入水 $(15 \mathrm{~mL})$, 乙酸乙酯萃取 $(15 \mathrm{~mL} \times 3)$, 合并有机相, 饱和食盐水洗 涤, 无水 $\mathrm{Na}_{2} \mathrm{SO}_{4}$ 干燥, 过滤, 旋蒸, 柱层色谱分离提纯 得到目标产物 $\mathbf{2 a} \sim 2 \mathbf{2 n}$ 及 $\mathbf{3 a}$.

$N$-芐基- $N$-对甲苯磺酰基-2-苯基-2-脄基乙酰胺(2a): 白色固体, m.p. 88 90 ${ }^{\circ} \mathrm{C}$ (文献值 ${ }^{[19]}$ : 89 91 ${ }^{\circ} \mathrm{C}$ ) ; ${ }^{1} \mathrm{H}$ NMR (400 MHz, $\left.\mathrm{CDCl}_{3}\right) \delta: 7.89(\mathrm{~d}, J=7.4 \mathrm{~Hz}, 2 \mathrm{H}), 7.75$ (d, $J=8.3 \mathrm{~Hz}, 2 \mathrm{H}), 7.62$ (t, $J=7.4 \mathrm{~Hz}, 1 \mathrm{H}), 7.49$ (t, $J=7.7$ $\mathrm{Hz}, 2 \mathrm{H}), 7.23$ (t, $J=6.6 \mathrm{~Hz}, 7 \mathrm{H}), 4.99(\mathrm{~s}, 2 \mathrm{H}), 2.40$ (s, $3 \mathrm{H}) ;{ }^{13} \mathrm{C}$ NMR (101 MHz, $\left.\mathrm{CDCl}_{3}\right) \delta: 189.0,168.8,146.9$, $135.9,135.8,135.7,134.2,131.1,130.9,130.1,129.8$, 129.3, 129.1, 49.6, 22.9.

$\mathrm{N}$-对甲氧基苄基- $\mathrm{N}$-对甲苯磺酰基-2-苯基-2-羰基乙 酰胺(2b): 白色固体, m.p. $135 \sim 137{ }^{\circ} \mathrm{C} ;{ }^{1} \mathrm{H}$ NMR (400
$\left.\mathrm{MHz}, \mathrm{CDCl}_{3}\right) \delta: 7.86(\mathrm{~d}, J=7.6 \mathrm{~Hz}, 2 \mathrm{H}), 7.76$ (d, $J=8.0$ $\mathrm{Hz}, 2 \mathrm{H}), 7.61$ (t, $J=7.2 \mathrm{~Hz}, 1 \mathrm{H}), 7.48(\mathrm{t}, J=7.5 \mathrm{~Hz}, 2 \mathrm{H})$, 7.24 (dd, $J=20.3,8.2 \mathrm{~Hz}, 4 \mathrm{H}), 6.76(\mathrm{~d}, J=8.3 \mathrm{~Hz}, 2 \mathrm{H})$, $4.90(\mathrm{~s}, 2 \mathrm{H}), 3.76(\mathrm{~s}, 3 \mathrm{H}), 2.41(\mathrm{~s}, 3 \mathrm{H}) ;{ }^{13} \mathrm{C}$ NMR $(101$ $\left.\mathrm{MHz}, \mathrm{CDCl}_{3}\right) \delta: 189.0,168.9,160.6,146.8,136.0,135.6$, $134.2,131.1,131.1,130.9,130.1,129.7,128.0,115.1$, 56.5, 49.2, 22.9; IR (KBr) v: 3062, 3003, 2969, 1677, 1610, 1597, 1363, 1214, 1165, 1091, $721 \mathrm{~cm}^{-1}$; HRMS (ESI) calcd for $\mathrm{C}_{23} \mathrm{H}_{21} \mathrm{NNaO}_{5} \mathrm{~S}(\mathrm{M}+\mathrm{Na})^{+}$446.1033, found 446.1038 .

$N$-邻溴苄基- $N$-对甲苯磺酰基-2-苯基-2-羰基乙酰胺 (2c): 白色固体, m.p. $158 \sim 159{ }^{\circ} \mathrm{C} ;{ }^{1} \mathrm{H}$ NMR $(400 \mathrm{MHz}$, $\left.\mathrm{CDCl}_{3}\right) \delta: 7.98(\mathrm{~d}, J=7.6 \mathrm{~Hz}, 2 \mathrm{H}), 7.76(\mathrm{~d}, J=8.2 \mathrm{~Hz}$, 2H), $7.66(\mathrm{t}, J=7.4 \mathrm{~Hz}, 1 \mathrm{H}), 7.54(\mathrm{t}, J=7.6 \mathrm{~Hz}, 2 \mathrm{H}), 7.45$ $(\mathrm{d}, J=7.9 \mathrm{~Hz}, 1 \mathrm{H}), 7.24(\mathrm{~d}, J=8.2 \mathrm{~Hz}, 2 \mathrm{H}), 7.16 \sim 7.03$ (m, 2H), $7.06(\mathrm{dd}, J=10.0,4.4 \mathrm{~Hz}, 1 \mathrm{H}), 5.11(\mathrm{~s}, 2 \mathrm{H}), 2.39$ $(\mathrm{s}, 3 \mathrm{H}) ;{ }^{13} \mathrm{C}$ NMR $\left(101 \mathrm{MHz}, \mathrm{CDCl}_{3}\right) \delta: 189.0,168.6$, $147.0,135.8,135.3,134.8,134.0,131.2,131.1,130.3$, 130.2, 129.8, 129.1, 128.7, 124.0, 49.2, 22.9; IR (KBr) v: 3062, 3027, 2967, 1674, 1596, 1375, 1218, 1180, 1088, $744 \mathrm{~cm}^{-1}$; HRMS (ESI) calcd for $\mathrm{C}_{22} \mathrm{H}_{18} \mathrm{BrNNaO}_{4} \mathrm{~S}(\mathrm{M}+$ $\mathrm{Na})^{+}$494.0032, found 494.0043 .

$N$-烯丙基- $N$-对甲苯磺酰基-2-苯基-2-羰基乙酰胺 (2d): 白色固体, m.p. $99 \sim 101{ }^{\circ} \mathrm{C}$ (文献值 ${ }^{[22 b]}: 98 \sim$ $\left.99{ }^{\circ} \mathrm{C}\right) ;{ }^{1} \mathrm{H}$ NMR $\left(400 \mathrm{MHz}, \mathrm{CDCl}_{3}\right) \delta: 7.94 \sim 7.89(\mathrm{~m}$, $4 \mathrm{H}), 7.66 \sim 7.62(\mathrm{~m}, 1 \mathrm{H}), 7.53(\mathrm{t}, J=7.7 \mathrm{~Hz}, 2 \mathrm{H}), 7.37(\mathrm{~d}$, $J=8.3 \mathrm{~Hz}, 2 \mathrm{H}), 5.75$ (ddt, $J=16.2,10.3,5.9 \mathrm{~Hz}, 1 \mathrm{H}), 5.20$ (ddd, $J=13.7,11.2,1.0 \mathrm{~Hz}, 2 \mathrm{H}), 4.35$ (d, $J=5.9 \mathrm{~Hz}, 2 \mathrm{H}$ ), $2.46(\mathrm{~s}, 3 \mathrm{H}) ;{ }^{13} \mathrm{C}$ NMR $\left(101 \mathrm{MHz}, \mathrm{CDCl}_{3}\right) \delta: 189.0,168.2$, $147.1,135.7,134.1,132.0,131.3,131.0,130.1,129.8$, 120.7, 48.5, 23.0 .

$\mathrm{N}$-正丁基- $\mathrm{N}$-对甲苯磺酰基-2-苯基-2-羰基乙酰胺 (2e): 白色固体, m.p. $85 \sim 87{ }^{\circ} \mathrm{C} ;{ }^{1} \mathrm{H}$ NMR $(400 \mathrm{MHz}$, $\left.\mathrm{CDCl}_{3}\right) \delta: 7.92(\mathrm{t}, J=7.9 \mathrm{~Hz}, 4 \mathrm{H}$ ), 7.64 (ddd, $J=8.6,2.3$, $1.2 \mathrm{~Hz}, 1 \mathrm{H}), 7.53(\mathrm{t}, J=7.6 \mathrm{~Hz}, 2 \mathrm{H}), 7.38(\mathrm{~d}, J=8.1 \mathrm{~Hz}$, $2 \mathrm{H}), 3.64$ (t, $J=8.0 \mathrm{~Hz}, 2 \mathrm{H}), 2.46$ (s, 3H), 1.66 (dt, $J=$ $11.9,7.7 \mathrm{~Hz}, 2 \mathrm{H}), 1.35 \sim 1.29(\mathrm{~m}, 2 \mathrm{H}), 0.91$ (t, $J=7.4 \mathrm{~Hz}$, $3 \mathrm{H}) ;{ }^{13} \mathrm{C}$ NMR $\left(101 \mathrm{MHz}, \mathrm{CDCl}_{3}\right) \delta: 189.2,168.6,147.0$, 135.8, 135.6, 134.2, 131.3, 130.9, 130.1, 129.7, 46.8, 31.5, 23.0, 21.3, 14.8; IR (KBr) v: 3075, 3031, 2959, 2871, 1676, 1598, 1367, 1214, 1163, 1089, $916 \mathrm{~cm}^{-1}$; HRMS (ESI) calcd for $\mathrm{C}_{19} \mathrm{H}_{21} \mathrm{NNaO}_{4} \mathrm{~S}(\mathrm{M}+\mathrm{Na})^{+}$382.1083, found 382.1088.

$N$-对氟芐基- $N$-对甲苯磺酰基-2-苯基-2-羰基乙酰胺 (2f): 白色固体, m.p. $93 \sim 95{ }^{\circ} \mathrm{C} ;{ }^{1} \mathrm{H}$ NMR $(400 \mathrm{MHz}$, 
$\left.\mathrm{CDCl}_{3}\right) \delta: 7.85(\mathrm{dd}, J=5.2,3.3 \mathrm{~Hz}, 2 \mathrm{H}), 7.76(\mathrm{~d}, J=8.4$ $\mathrm{Hz}, 2 \mathrm{H}), 7.62$ (ddd, $J=8.7,2.5,1.2 \mathrm{~Hz}, 1 \mathrm{H}), 7.49$ (dd, $J=$ $10.7,4.8 \mathrm{~Hz}, 2 \mathrm{H}), 7.28 \sim 7.25(\mathrm{~m}, 4 \mathrm{H}), 6.94 \sim 6.90(\mathrm{~m}$, $2 \mathrm{H}), 4.93(\mathrm{~s}, 2 \mathrm{H}), 2.41(\mathrm{~s}, 3 \mathrm{H}) ;{ }^{13} \mathrm{C} \mathrm{NMR}(101 \mathrm{MHz}$, $\left.\mathrm{CDCl}_{3}\right) \delta: 189.0,168.8,163.7(\mathrm{~d}, J=245.0 \mathrm{~Hz}), 147.1$, $135.8,134.1,131.9,131.8,131.5$ (d, $J=8.0 \mathrm{~Hz}), 131.1$, $130.9,130.1,129.7,116.7$ (d, $J=22.0 \mathrm{~Hz}), 48.9,22.9$; IR (KBr) v: 3053, 2986, 1687, 1664, 1595, 1357, 1212, 1167, 1088, $717 \mathrm{~cm}^{-1}$; HRMS (ESI) calcd for $\mathrm{C}_{22} \mathrm{H}_{18} \mathrm{FNNaO}_{4} \mathrm{~S}$ $(\mathrm{M}+\mathrm{Na})^{+}$434.0833, found434.0834.

1-苯基-2-[3-(噁唑啉-2-酮)基]-1,2-二酮(2g): 白色固 体, m.p. $144 \sim 146{ }^{\circ} \mathrm{C}$ (文献值 ${ }^{[20]}: 149 \sim 150{ }^{\circ} \mathrm{C}$ ); ${ }^{1} \mathrm{H}$ NMR (400 MHz, $\left.\mathrm{CDCl}_{3}\right) \delta: 7.87(\mathrm{~d}, J=7.5 \mathrm{~Hz}, 2 \mathrm{H}), 7.63$ $(\mathrm{t}, J=7.4 \mathrm{~Hz}, 1 \mathrm{H}), 7.50(\mathrm{t}, \quad J=7.7 \mathrm{~Hz}, 2 \mathrm{H}), 4.56(\mathrm{t}, J=$ $8.0 \mathrm{~Hz}, 2 \mathrm{H}), 4.13(\mathrm{t}, J=8.0 \mathrm{~Hz}, 2 \mathrm{H}) ;{ }^{13} \mathrm{C} \mathrm{NMR}(101 \mathrm{MHz}$, $\left.\mathrm{CDCl}_{3}\right) \delta: 186.8,165.4,152.1,133.8,131.3,128.3,128.0$, 63.0, 39.9 .

$N$-对甲氧基苄基- $N$-甲磺酰基-2-苯基-2-羰基乙酰胺 (2h): 白色固体, m.p. 104 106 ${ }^{\circ} \mathrm{C} ;{ }^{1} \mathrm{H}$ NMR (400 MHz, $\left.\mathrm{CDCl}_{3}\right) \delta: 7.89(\mathrm{~d}, J=7.3 \mathrm{~Hz}, 2 \mathrm{H}), 7.63 \sim 7.61(\mathrm{~m}, 1 \mathrm{H})$, 7.50 (t, $J=6.9 \mathrm{~Hz}, 2 \mathrm{H}), 7.38$ (d, $J=7.4 \mathrm{~Hz}, 2 \mathrm{H}), 6.92$ (d, $J=7.4 \mathrm{~Hz}, 2 \mathrm{H}), 5.10$ (s, 2H), 3.81 (s, 3H), 2.88 (s, 3H); ${ }^{13} \mathrm{C}$ NMR (101 MHz, $\mathrm{CDCl}_{3}$ ) $\delta: 188.5,167.1,159.8,134.7$, 132.3, 130.4, 129.9, 128.8, 126.7, 114.4, 55.3, 47.1, 43.1; IR (KBr) v: 3024, 3007, 2955, 1686, 1676, 1613, 1514, 1354, 1214, 1051, $898 \mathrm{~cm}^{-1}$; HRMS (ESI) calcd for $\mathrm{C}_{17} \mathrm{H}_{17} \mathrm{NNaO}_{5} \mathrm{~S}(\mathrm{M}+\mathrm{Na})^{+}$370.0720, found 370.0723 .

$N$-苠基- $N$-甲磺酰基-2-苯基-2-羰基乙酰胺(2i): 白 色固体, m.p. $118 \sim 120{ }^{\circ} \mathrm{C} ;{ }^{1} \mathrm{H} \mathrm{NMR}\left(400 \mathrm{MHz}, \mathrm{CDCl}_{3}\right) \delta$ : $7.90(\mathrm{~d}, J=7.3 \mathrm{~Hz}, 2 \mathrm{H}), 7.63(\mathrm{t}, J=6.5 \mathrm{~Hz}, 1 \mathrm{H}), 7.50$ (t, $J=6.9 \mathrm{~Hz}, 2 \mathrm{H}), 7.43 \sim 7.36(\mathrm{~m}, 5 \mathrm{H}), 5.16(\mathrm{~s}, 2 \mathrm{H}), 2.90(\mathrm{~s}$, $3 \mathrm{H}) ;{ }^{13} \mathrm{C}$ NMR (101 MHz, $\left.\mathrm{CDCl}_{3}\right) \delta: 188.5,167.1,134.7$, 132.3, 130.0, 129.1, 128.8, 128.7, 128.6, 47.5, 43.0; IR (KBr) v: 3076, 3025, 2934, 1678, 1597, 1510, 1359, 1224, 1064, $716 \mathrm{~cm}^{-1}$; HRMS (ESI) calcd for $\mathrm{C}_{16} \mathrm{H}_{15} \mathrm{NNaO}_{4} \mathrm{~S}$ $(\mathrm{M}+\mathrm{Na})^{+}$340.0614, found 340.0615 .

$N$-对氟芳基- $N$-甲磺酰基-2-苯基-2-羰基乙酰胺 $(2 \mathbf{j})$ : 白色固体, m.p. $143 \sim 144{ }^{\circ} \mathrm{C} ;{ }^{1} \mathrm{H}$ NMR (400 MHz, $\mathrm{CDCl}_{3}$ ) $\delta: 7.88(\mathrm{~d}, J=7.3 \mathrm{~Hz}, 2 \mathrm{H}), 7.64(\mathrm{t}, J=6.6 \mathrm{~Hz}, 1 \mathrm{H}), 7.52 \sim$ $7.44(\mathrm{~m}, 4 \mathrm{H}), 7.09(\mathrm{t}, J=7.8 \mathrm{~Hz}, 2 \mathrm{H}), 5.11(\mathrm{~s}, 2 \mathrm{H}), 2.96(\mathrm{~s}$, $3 \mathrm{H}) ;{ }^{13} \mathrm{C} \mathrm{NMR}\left(101 \mathrm{MHz}, \mathrm{CDCl}_{3}\right) \delta: 188.4,167.1,162.8$ $(\mathrm{d}, J=247.0 \mathrm{~Hz}), 134.8,132.2,130.8$ (d, $J=8.0 \mathrm{~Hz})$, 129.9, 128.8, 116.1 (d, $J=22.0 \mathrm{~Hz}), 46.9$, 43.1; IR (KBr) $v: 3076,3035,2934,1678,1597,1510,1358,1224,1160$, 1064, $716 \mathrm{~cm}^{-1}$; HRMS (ESI) calcd for $\mathrm{C}_{16} \mathrm{H}_{14} \mathrm{FNNaO}_{4} \mathrm{~S}$
$(\mathrm{M}+\mathrm{Na})^{+}$358.0520, found 358.0521.

$N$-对甲苄基- $N$-甲磺酰基-2-苯基-2-羰基乙酰胺(2k): 白色固体, m.p. $126 \sim 128{ }^{\circ} \mathrm{C} ;{ }^{1} \mathrm{H}$ NMR (400 MHz, $\mathrm{CDCl}_{3}$ ) $\delta: 7.90(\mathrm{~d}, J=7.5 \mathrm{~Hz}, 2 \mathrm{H}), 7.65 \sim 7.62(\mathrm{~m}, 1 \mathrm{H}), 7.50(\mathrm{t}$, $J=7.0 \mathrm{~Hz}, 2 \mathrm{H}), 7.33(\mathrm{~d}, J=7.1 \mathrm{~Hz}, 2 \mathrm{H}), 7.22(\mathrm{t}, J=11.9$ $\mathrm{Hz}, 2 \mathrm{H}), 5.12$ (s, 2H), 2.89 (s, 3H), 2.36 (s, 3H); ${ }^{13} \mathrm{C}$ NMR $\left(101 \mathrm{MHz}, \mathrm{CDCl}_{3}\right) \delta: 188.5,167.1,138.5,134.7,132.3$, $131.7,129.9,129.7,128.8,128.7,47.3,43.1,21.2$; IR (KBr) v: 3025, 2922, 1678, 1598, 1356, 1208, 1156, 1046, $715 \mathrm{~cm}^{-1}$; HRMS (ESI) calcd for $\mathrm{C}_{17} \mathrm{H}_{17} \mathrm{NNaO}_{4} \mathrm{~S}(\mathrm{M}+$ $\mathrm{Na})^{+}$354.0770, found 354.0769.

$N$-邻氯茮基- $N$-甲磺酰基-2-苯基-2-羰基乙酰胺(2l): 白色固体, m.p. $110 \sim 111{ }^{\circ} \mathrm{C} ;{ }^{1} \mathrm{H}$ NMR (400 MHz, $\mathrm{CDCl}_{3}$ ) $\delta: 7.94(\mathrm{~d}, J=7.5 \mathrm{~Hz}, 2 \mathrm{H}), 7.67$ (dd, $J=21.9,15.4 \mathrm{~Hz}$, $1 \mathrm{H}), 7.53 \sim 7.45(\mathrm{~m}, 4 \mathrm{H}), 7.28(\mathrm{~d}, J=25.7 \mathrm{~Hz}, 2 \mathrm{H}), 5.24$ $(\mathrm{s}, 2 \mathrm{H}), 3.03(\mathrm{~s}, 3 \mathrm{H}) ;{ }^{13} \mathrm{C} \mathrm{NMR}\left(101 \mathrm{MHz}, \mathrm{CDCl}_{3}\right) \delta$ : $188.0,166.8,134.8,133.6,132.3,132.0,130.1,130.0$, 129.6, 129.6, 128.8, 127.2, 45.6, 42.6; IR (KBr) v: 3060, 3014, 2965, 1682, 1597, 1367, 1205, 1159, 1050, 708 $\mathrm{cm}^{-1}$; HRMS (ESI) calcd for $\mathrm{C}_{16} \mathrm{H}_{14} \mathrm{CINNaO}_{4} \mathrm{~S}(\mathrm{M}+\mathrm{Na})^{+}$ 374.0224, found 374.0227.

$N$-对甲氧基苯基- $N$-甲磺酰基-2-苯基-2-羰基乙酰胺 (2m): 白色固体, m.p. 138 140 ${ }^{\circ} \mathrm{C} ;{ }^{1} \mathrm{H}$ NMR (400 MHz, $\left.\mathrm{CDCl}_{3}\right) \delta: 7.93(\mathrm{~d}, J=7.1 \mathrm{~Hz}, 2 \mathrm{H}), 7.66(\mathrm{t}, J=17.5 \mathrm{~Hz}$, $1 \mathrm{H}), 7.51$ (t, $J=6.9 \mathrm{~Hz}, 2 \mathrm{H}), 7.27$ (d, $J=7.9 \mathrm{~Hz}, 2 \mathrm{H}), 6.94$ $(\mathrm{d}, J=7.6 \mathrm{~Hz}, 2 \mathrm{H}), 3.81(\mathrm{~s}, 3 \mathrm{H}), 3.40(\mathrm{~s}, 3 \mathrm{H}) ;{ }^{13} \mathrm{C} \mathrm{NMR}$ $\left(101 \mathrm{MHz}, \mathrm{CDCl}_{3}\right) \delta: 188.0,167.3,160.9,134.9,132.2$, $131.2,129.8,128.9,125.6,115.0,55.5,41.0$; IR (KBr) $v$ : 3068, 3015, 2979, 1685, 1608, 1508, 1367, 1239, 1150, 1028, $708 \mathrm{~cm}^{-1}$; HRMS (ESI) calcd for $\mathrm{C}_{16} \mathrm{H}_{15} \mathrm{NNaO}_{5} \mathrm{~S}$ $(\mathrm{M}+\mathrm{Na})^{+}$356.0563, found 356.0568.

$N$-对甲苯基- $N$-甲磺酰基-2-苯基-2-羰基乙酰胺(2n): 白色固体, m.p. 127 $128{ }^{\circ} \mathrm{C} ;{ }^{1} \mathrm{H}$ NMR (400 MHz, $\mathrm{CDCl}_{3}$ ) $\delta$ : $7.94(\mathrm{~d}, J=7.2 \mathrm{~Hz}, 2 \mathrm{H}), 7.66$ (dd, $J=20.8,14.4 \mathrm{~Hz}$, $1 \mathrm{H}), 7.51(\mathrm{t}, J=7.5 \mathrm{~Hz}, 2 \mathrm{H}), 7.25(\mathrm{~s}, 4 \mathrm{H}), 3.40(\mathrm{~s}, 3 \mathrm{H})$, $2.38(\mathrm{~s}, 3 \mathrm{H}) ;{ }^{13} \mathrm{C}$ NMR $\left(101 \mathrm{MHz}, \mathrm{CDCl}_{3}\right) \delta: 188.0,167.2$, $140.8,134.9,132.2,130.7,130.5,129.9,129.7,128.9$, 41.1, 21.3; IR (KBr) v: 3016, 2932, 1691, 1610, 1598, 1507, 1368, 1236, 1158, 1023, $710 \mathrm{~cm}^{-1}$; HRMS (ESI) calcd for $\mathrm{C}_{16} \mathrm{H}_{15} \mathrm{NNaO}_{4} \mathrm{~S}(\mathrm{M}+\mathrm{Na})^{+}$340.0614, found 340.0615 .

5-苯基-3-对甲苯基噁唑-2-酮(3a): 白色固体, m.p. $181 \sim 183{ }^{\circ} \mathrm{C} ;{ }^{1} \mathrm{H}$ NMR $\left(400 \mathrm{MHz}, \mathrm{CDCl}_{3}\right) \delta: 7.55 \sim 7.54$ $(\mathrm{m}, 2 \mathrm{H}), 7.48(\mathrm{~d}, J=8.5 \mathrm{~Hz}, 2 \mathrm{H}), 7.41 \sim 7.38(\mathrm{~m}, 2 \mathrm{H})$, $7.34 \sim 7.28(\mathrm{~m}, 1 \mathrm{H}), 7.27 \sim 7.21(\mathrm{~m}, 2 \mathrm{H}), 7.12(\mathrm{~s}, 1 \mathrm{H})$, 
$2.36(\mathrm{~s}, 3 \mathrm{H}) ;{ }^{13} \mathrm{C}$ NMR $\left(101 \mathrm{MHz}, \mathrm{CDCl}_{3}\right) \delta: 154.0,141.0$, $137.9,134.3,131.3,130.2,129.7,128.3,124.4,122.3$, 110.0, 22.2; IR (KBr) v: 3127, 3037, 1742, 1655, 1517, 1398, 1250, 1207, 1056, 1024, $734 \mathrm{~cm}^{-1}$; HRMS (ESI) calcd for $\mathrm{C}_{16} \mathrm{H}_{13} \mathrm{NNaO}_{2}(\mathrm{M}+\mathrm{Na})^{+}$274.0838, found 274.0838 .

辅助材料(Supporting Information) 合成产物的 ${ }^{1} \mathrm{H}$ $\mathrm{NMR}$ 及 ${ }^{13} \mathrm{C} \mathrm{NMR}$ 谱图. 这些材料可以免费从本刊网站 (http://sioc-journal.cn/)上下载

\section{References}

[1] Kher, S. S.; Penzo, M.; Fulle, S.; Finn, P. W.; Blackman, M. J.; Jirgensons, A. Bioorg. Med. Chem. Lett. 2014, 24, 4486.

[2] (a) Korukonda, R.; Guan, N.; Dalton, J. T.; Liu, J.; Donkor, I. O. J. Med. Chem. 2006, 49, 5282

(b) Donkor, I. O.; Han, J.; Zheng, X. J. Med. Chem. 2004, 47, 72.

(c) Yoo, Y. J.; Nam, D. H.; Jung, S. Y.; Jang, J. W.; Kim, H. J.; Jin, C.; Pae, A. N.; Lee, Y. S. Bioorg. Med. Chem. Lett. 2011, 21, 2850. (d) Ovat, A.; Li, Z. Z.; Hampton, C. Y.; Asress, S. A.; Fernández, F. M.; Glass, J. D.; Powers, J. C. J. Med. Chem. 2010, 53, 6326.

[3] Mandadapu, S. R.; Weerawarna, P. M.; Gunnam, M. R.; Alliston, K. R.; Lushington, G. H.; Kim, Y.; Chang, K.-O.; Groutas, W. C. Bioorg. Med. Chem. Lett. 2012, 22, 4820.

[4] Steuer, C.; Gege, C.; Fischl, W.; Heinonen, K. H.; Bartenschlager, R.; Klein, C. D. Bioorg. Med. Chem. 2011, 19, 4067.

[5] Chen, J.-C.; Uang, B.-J.; Lyu, P.-C.; Chang, J.-Y.; Liu, K.-J.; Kuo, C.-C.; Hsieh, H.-P.; Wang, H.-C.; Cheng, C.-S.; Chang, Y.-H.; Chang, M. D.-T.; Chang, W.-S. W.; Lin, C.-C. J. Med. Chem. 2010, $53,4545$.

[6] Chiou, A.; Markidis, T.; Constantinou-Kokotou, V.; Verger, R.; KoKotos, G. Org. Lett. 2000, 2, 347.

[7] Bennett, F.; Huang, Y.; Hendrata, S.; Lovey, R.; Bogen, S. L.; Pan, W.; Guo, Z.; Prongay, A.; Chen, K. X.; Arasappan, A.; Venkatraman, S.; Velazquez, F.; Nair, L.; Sannigrahi, M.; Tong, X.; Pichardo, J.; Cheng, K.-C.; Girijavallabhan, V. M.; Saksena, A. K.; Njoroge, F. G. Bioorg. Med. Chem. Lett. 2010, 20, 2617.

[8] Lin, C.; Kwong, A. D.; Perni, R. B. Infect. Disord.: Drug Targets 2006, $6,3$.

[9] Schnopp, C.; Remling, R.; Möhrenschlager, M.; Weigl, L.; Ring, J.; Abeck, D. J. Am. Acad. Dermtol. 2002, 46, 73.

[10] (a) Stella, S.; Chadha, A. Catal. Today 2012, 198, 345.

(b) Singh, R. P.; Shreeve, J. M. J. Org. Chem. 2003, 68, 6063.

(c) Guin, S.; Rout, S. K.; Gogoi, A.; Ali, W.; Patel, B. K. Adv. Synth. Catal. 2014, 356, 2559.

(d) Zhang, X.; Yang, W.; Wang, L. Org. Biomol. Chem. 2013, 11, 3649 .

(e) Wang, H.; Guo, L.-N.; Duan, X.-H. Org. Biomol. Chem. 2013, 11, 4573 .

[11] (a) Du, F.-T.; Ji, J.-X. Chem. Sci. 2012, 3, 460.

(b) Mai, W.-P.; Wang, H.-H.; Li, Z.-C.; Yuan, J.-W.; Xiao, Y.-M.; Yang, L.-R.; Mao, P.; Qu, L.-B. Chem. Commun. 2012, 48, 10117.

(c) Zhang, X.; Wang, L. Green Chem. 2012, 14, 2141.

(d) Zhang, J.; Wei, Y.; Lin, S.; Liang, F.; Liu, P. Org. Biomol. Chem. 2012, 10, 9237.

(e) Zhang, Z.; Su, J.; Zha, Z.; Wang, Z. Chem. Commun. 2013, 49, 8982 .

(f) Zhao, Q.; Miao, T.; Zhang, X.; Zhou, W.; Wang, L. Org. Biomol.
Chem. 2013, 11, 1867.

(g) Liu, S.; Gao, Q.; Wu, X.; Zhang, J.; Ding, K.; Wu, A. Org. Biomol. Chem. 2015, 13, 2239.

(h) Wei, W.; Shao, Y.; Hu, H.; Zhang, F.; Zhang, C.; Xu, Y.; Wan, X. J. Org. Chem. 2012, 77, 7157.

(i) Gong, J.; Zhu, Z.; Lu, L.; Guo, S.; Cai, H. Chin. J. Org. Chem. 2015, 35, 1917 (in Chinese).

(龚久涵, 朱正, 卢林, 郭生梅, 蔡琥, 有机化学, 2015, 35, 1917.)

[12] (a) Deshidi, R.; Kumar, M.; Devari, S.; Shah, B. A. Chem. Commun. 2014, 50, 9533.

(b) Sagadevan, A.; Ragupathi, A.; Lin, C.-C.; Hwu, J. R.; Hwang, K. C. Green Chem. 2015, 17, 1113.

(c) Ren, X.; Wang, G.; Tang, X.; Zhao, W. Chin. J. Org. Chem. 2015, 35, 1733 (in Chinese).

(任相伟, 王光伟, 唐向阳, 赵温涛, 有机化学, 2015, 35, 1733.)

[13] Dutta, S.; Kotha, S. S.; Sekar, G. RSC Adv. 2015, 5, 47265.

[14] Du, B.; Jin, B.; Sun, P. Org. Biomol. Chem. 2014, 12, 4586.

[15] (a) Zhang, C.; Zong, X.; Zhang, L.; Jiao, N. Org. Lett. 2012, 14, 3280 .

(b) Mupparapu, N.; Khan, S.; Battula, S.; Kushwaha, M.; Gupta, A. P.; Ahmed, Q. N.; Vishwakarma, R. A. Org. Lett. 2014, 16, 1152.

[16] Zhang, C.; Xu, Z.; Zhang, L.; Jiao, N. Angew. Chem., Int. Ed. 2011, 50,11088 .

[17] (a) Takács, E.; Varga, C.; Skoda-Földes, R.; Kollár, L. Tetrahedron Lett. 2007, 48, 2453.

(b) Papp, M.; Skoda-Földes, R. J. Mol. Catal. A 2013, 378, 193.

(c) Du, H.; Ruan, Q.; Qi, M.; Han, W. J. Org. Chem. 2015, 80, 7816.

(d) Genelot, M.; Villandier, N.; Bendjeriou, A.; Jaithong, P.; Djakovitch, L.; Dufaud, V. Catal. Sci. Technol. 2012, 2, 1886.

(e) Zhang, X.; Yang, C.-L.; Song, W.; Zhang, Y.-L. J. Mol. Catal. (China) 2014, 28, 312 (in Chinese).

(张欣，杨彩玲，宋伟伟，张远丽，刘建明，卓克垒，分子催化， 2014, 28, 312.)

(f) Zhang, N.; Zhang, Y.-L.; Liu, G.; Zhang, X.; Wang, Y.-L.; Liu, J.; Zhou, K. J. Mol. Catal. (China) 2013, 27, 250 (in Chinese).

(张宁飞, 张远丽, 刘规划, 张欣, 王永丽, 刘建明, 卓克垒, 分 子催化, 2013, 27, 250.)

[18] Xing, Q.; Shi, L.; Lang, R.; Xia, C.; Li, F. Chem. Commun. 2012, $48,11023$.

[19] Al-Rashid, Z. F.; Johnson, W. L.; Hsung, R. P.; Wei, Y.; Yao, P.-Y.; Liu, R.; Zhao, K. J. Org. Chem. 2008, 73, 8780.

[20] Huang, H.; He, G.; Zhu, X.; Jin, X.; Qiu, S.; Zhu, H. Eur. J. Org. Chem. 2014, 7174 .

[21] Chikugo, T.; Yauchi, Y.; Ide, M.; Iwasawa, T. Tetrahedron 2014, 70, 3988.

[22] (a) Xu, C.-F.; Xu, M.; Jia, Y.-X.; Li, C.-Y. Org. Lett. 2011, 13, 1556.

(b) Wang, K.-B.; Ran, R.-Q.; Xiu, S.-D.; Li, C.-Y. Org. Lett. 2013, $15,2374$.

[23] (a) Zuo, J.-F.; Huang, W.-S.; Li, L.; Xu, Z.; Zheng, Z.-J.; Yang, K.-F.; Xu, L.-W. RSC Adv. 2015, 5, 30389.

(b) Liu, F.-L.; Chen, J.-R.; Zuo, Y.-Q.; Wei, Q.; Xiao, W.-J. Org. Lett. 2014, 16, 3768.

(c) Le, H. V.; Ganem, B. Org. Lett. 2011, 13, 2584.

(d) Vieira, A. A.; Azeredo, J. B.; Godoi, M.; Santi, C.; Júnior, E. N. S.; Braga, A. L. J. Org. Chem. 2015, 80, 2120.

(e) Prasad, P. K.; Reddi, R. N.; Sudalai, A. Org. Lett. 2016, $18,500$.

[24] Gao, A.; Yang, F.; Li, J.; Wu, Y. Tetrahedron 2012, 68, 4950.

[25] (a) Dekorver, K. A.; Hsung, R. P.; Song, W.-Z.; Wang, X.-N.; Walton, M. C. Org. Lett. 2012, 14, 3214.

(b) Wang, X.-N.; Winston-Mcpherson, G. N.; Walton, M. C.; Zhang, 
Y.; Hsung, R. P.; Dekorver, K. A. J. Org. Chem. 2013, 78, 6233.

(c) Dekorver, K. A.; Johnson, W. L.; Zhang, Y.; Hsung, R. P.; Dai, H.; Deng, J.; Lohse, A. G.; Zhang, Y.-S. J. Org. Chem. 2011, 76, 5092.

[26] (a) Huang, H.; He, G.; Zhu, G.; Zhu, X.; Qiu, S.; Zhu, H. J. Org. Chem. 2015, 80, 3480 .

(b) Istrate, F. M.; Buzas, A. K.; Jurberg, I. D.; Odabachian, Y.; Gagosz, F. Org. Lett. 2008, 10, 925.

(c) Lu, Z.; Cui, W.; Xia, S.; Bai, Y.; Luo, F.; Zhu, G. J. Org. Chem. 2012, 77, 9871. (d) Lu, Z.; Xu, X.; Yang, Z.; Kong, L.; Zhu, G. Tetrahedron Lett. 2012, 53, 3433.

(e) Hashmi, A. S. K.; Salathé, R.; Frey, W. Synlett 2007, 1763.

[27] (a) Métayer, B.; Compain, G.; Jouvin, K.; Martin-Mingot, A.; Bachmann, C.; Marrot, J.; Evano, G.; Thibaudeau, S. J. Org. Chem. 2015, 80, 3397.

(b) Meng, T.; Chen, R.; Liu, L.; Wang, T.; Liu, X.; Zhao, W. Chin. J. Org. Chem. 2015, 35, 2108 (in Chinese).

(孟团结, 陈荣祥, 刘澜涛, 王涛, 刘新明, 赵文献, 有机化学, 2015, 35, 2108.)

(Zhao, X.) 